\title{
Stochastic Approach
}

\author{
H. - P. Gail \\ Institut für Theoretische Astrophysik \\ der Universität Heidelberg \\ Heidelberg, FRG
}

\section{Abstract}

A general formalism for describing the radiation transfer in a medium with arbitrary velocity fields is presented. It is demonstrated that classical microturbulence and mesoturbulent models based on Markov processes can be considered as the two lowest order members within a hierarchy of model equations with an increasing degree of approximation to reality. Some preliminary results concerning the relevance of low order model equations are presented.

\section{Introduction}

In interpreting line profiles originating from stellar atmospheres with internal motions, one encounters the problem that the observed flux within a spectral line is composed of contributions from regions with quite different velocities. In order to obtain the line profile which actually is observed, an averaging process has to be applied with respect to the ensemble of flow situations which occur along all lines of sight which contribute to the measured flux.

If the velocity distribution $\vec{v}(\vec{x}, t)$ within the atmosphere is known, the calculation of the radiation intensity exhibits no special problem (except for numerical difficulties) and the required average is simply calculated as an integral of the emergent intensity over all directions of interest. In this case, there is no need for a statistical treatment of the line transfer problem.

If, however, the flow is turbulent and thus can only be described in terms of its statistical properties or if the information on the state of motion of the matter is incomplete, it is not possible to assign in an unambiguous way to each line of sight a velocity profile $v(r)$ along this ray. On the other hand, a definitive knowledge of this function $v(r)$ is a pre-requisite for solving the ordinary equation of radiative transfer in a moving medium. In this case, one has to take recourse to statistical methods. 
Even if the statistical properties of the flow are completely known (for instance, if the complete hierarchy of probability densities defined in chapter 2 is known), it is not possible to specify uniquely the quantity $v(r)$ for each line of sight. However, for a large ensemble of equivalent rays it is possible to determine for each possible distribution of velocities along a ray the probability, that this specific $v(r)$ is realized. Since, for a given $\mathrm{v}(r)$, one is able to solve the radiative transfer problem, one obtains a certain intensity distribution $I(r)$ along the ray. The probability of realization of this distribution $I(r)$ equals the probability of realization of the specific $v(r)$, on which this solution $I(r)$ is based. Thus it is natural to describe the radiation field in terms of probability densities and to reformulate the radiative transfer equation in terms of these quantities. A general theory of this kind has been developped for the case of LTE and negligable scattering (see chapter 3 and 4 ).

If no or incomplete information about the velocity field is available, it is nevertheless useful to apply statistical methods. The best procedure in this case would be (i) to isolate the basic parameters of the velocity field which are relevant for the line transfer problem and (ii) to substitute the equations of the original problem by model equations, which depend on the relevant parameters only.

One method to proceed in this direction is, to derive a hierarchy of statistical model equations, which incorporate an increasing degree of information on the structure of the velocity field. By a study of the properties of the members of such a hierarchy it will be possible to find out the relevant parameters and the adequate model equations. The classical microturbulence model and the Markov-process models of Auvergne et al.(1973) and Gail et al.(1974) can be considered as the zeroth order and the first order models within such a hierarchy (see chapter 5). Model equations of higher order have not been derived up to now. Thus, it is not possible at present to decide, whether first order model equations already are sufficient to treat the line transfer problem or not.

A second method is to start with a statistical theory, valid for general velocity fields, and to derive from this the model equations. Some preliminary results in this direction are presented in chapter 6 .

\section{Description of the velocity field}

The ensemble of different flow situations, which one encounters along 
different rays in a stellar atmosphere is most conveniently described by the hierarchy of n-point probability densities

$\mathbb{P}_{n}\left(\vec{x}_{1}, \vec{v}_{1} ; \vec{x}_{2}, \vec{v}_{2} ; \ldots ; \vec{x}_{n}, \vec{v}_{n}\right)=\mathbb{P}_{n}(1, \ldots, n)$

$\mathbf{P}_{n}$ is the probability of finding at $\vec{x}_{1}$ the velocity $\vec{v}_{1}$ and at $\vec{x}_{2}$ the velocity $\vec{v}_{2} \ldots$ and at $\vec{x}_{n}$ the velocity $\vec{v}_{n}$. The following properties of the $P_{n}$ are self evident:

$\mathbb{P}_{n}(1, \ldots, n) \geq 0$,

$\int d^{3} \vec{v}_{i} \mathbb{P}_{n}(1, \ldots, i-1, i, i+1, \ldots, n)=\mathbb{P}_{n-1}(1, \ldots, i-1, i+1, \ldots, n)$,

$\int d^{3} v_{1} \mathbb{P}_{1}(1)=1$

Since only the component of the velocity parallel to the ray under consideration enters into the radiative transfer problem, we define a new probability density by

$$
\begin{aligned}
\mathbb{P}_{n}\left(x_{1}, v_{1 \|} ; x_{2}, v_{2 \|} ; \ldots ; x_{n}, v_{n \|} ; \vec{k}\right)= \\
\\
\int d^{2} v_{1 \perp} \ldots \int d^{2} v_{n \perp} \mathbb{P}_{n}(1, \ldots, n)
\end{aligned}
$$

which gives the corresponding probabilities for the $\|$ component of the velocity. For anisotropic $\mathbb{P}_{n}\left(\vec{x}_{1}, \vec{v}_{1} ; \ldots\right)$ they depend explicitly on the direction $\vec{k}$ of the ray. The $x_{i}(i=1, \ldots, n)$ are the coordinates of the points to which $\mathbb{P}_{n}$ refers along the ray. We assume these points to form an ordered sequence with $x_{1} \leq x_{2} \leq \cdots \leq x_{n}$, since in the application to the radiative transfer problem the $\mathbb{P}_{n}$ occur only in this special form. In the following we simply write $v_{i}$ instead of $v_{i l l}$ and omit the $\vec{k}$ from our notation.

Since every hydrodynamic flow has the property of being continuous for distances between the points $x_{i}, x_{i+1}$ smaller than a certain length, the probability densities $\mathbb{P}_{n}$ have to satisfy the condition:

$\lim \quad \mathbf{P}_{n}(1, \ldots, i, i+1, i+2, \ldots, n)=\mathbb{P}_{n-1}(1, \ldots, i, i+2, \ldots, n) \cdot \delta\left(v_{i-1}-v_{i}\right)$ $x_{i+1}+x_{i}$

since, if $x_{i+1}$ equals $x_{i}$ then $v_{i+1}$ necessarely equals $v_{i}$ due to the con- 
tinuity of the flow. Especially, it follows

$\lim _{x_{n} \rightarrow x_{1}} \mathbb{P}_{n}(1, \ldots, n)=\mathbb{P}_{1}(1) \prod_{i=2}^{n} \delta\left(v_{i-1}-v_{i}\right)$

A wide class of flows has the property, that there exists a finite correlation length 1 such that the velocities $v_{i+1}$ and $v_{i}$ become statistically independent, if the distance between $x_{i+1}$ and $x_{i}$ becomes large compared to 1 . If the flow has this special property, then the $\mathbb{P}_{n}$ 's have to satisfy the condition

$\mathbb{P}_{n}(1, \ldots, i, i+1, \ldots, n)=\mathbb{P}_{i}(1, \ldots, i) \mathbb{P}_{n-i}(i+1, \ldots, n)$ if $x_{i+1}-x_{i}>>1$.

This condition is valid for instance for turbulent flows. It is not valid for instance for harmonic waves.

The concept of a description of the velocity field by means of the hierarchy of the $\mathbf{P}_{n}$ 's is flexible enough to allow a unified description of such different cases like (for instance) a purely deterministic velocity field $v(x)$ :

$\mathbb{P}_{n}(1, \ldots, n)=\delta\left(v_{1}-v\left(x_{1}\right)\right) \cdot \delta\left(v_{2}-v\left(x_{2}\right)\right) \cdots \delta\left(v_{n}-v\left(x_{n}\right)\right)$

or a pure noise

$\mathbb{P}_{n}(1, \ldots, n)=\mathbb{P}_{1}\left(x_{1}, v_{1}\right) \cdots \mathbb{P}_{1}\left(x_{n}, v_{n}\right)$

\section{Description of the Radiation Field}

In analogy to the description of the velocity field by means of the $\mathbb{P}_{n}$ 's we describe the joint process $(I, V)$ by means of the hierarchy of $n$-point probability densities (Gail et al, 1979, henceforth called paper I):

$P_{n}\left(x_{1}, v_{1}, I_{1} ; x_{2}, v_{2}, I_{2} ; \ldots ; x_{n}, v_{n}, I_{n} ; \vec{k}, v\right)=P_{n}(1, \ldots, n)$

$P_{n}$ is the probability of finding at $x_{1}$ the velocity $v_{1}$ and the intensity of radiation $I_{1}$ and at $x_{2}$ the velocity $v_{2}$ and the intensity $I_{2} \ldots$ and at $x_{n}$ the velocity $v_{n}$ and the intensity $I_{n}$. From the physical pro- 
perties of radiative transfer it follows, that we are interested only in probability densities, where the $x_{i}(i=1, \ldots, n)$ form an ordered sequence along the ray under consideration (with direction $\vec{k}$ ).

The probability densities $P_{n}$ have to satisfy conditions analogue to $(2.2),(2.3)$ and $(2.4)$. In paper I it is shown, that the general $P_{n}$ can be written as

$P_{n}(1, \ldots, n)=P_{1}(1) \prod_{i=2}^{n-1} P_{2}(i \mid i+1) \mathbb{P}_{n}(1, \ldots, n) /\left(\mathbb{P}_{1}(1) \prod_{i=2}^{n-1} \mathbb{P}_{2}(i \mid i+1)\right)$.

Here we have introduced the conditional probabilities

$P_{2}(1 \mid 2)=P_{2}(1,2) / P_{1}(1)$

$\mathbf{P}_{2}(1 \mid 2)=\mathbb{P}_{2}(1,2) / \mathbb{P}_{1}(1)$

Hence, the complete information on the radiation field is already contained in $\mathrm{P}_{2}$.

The conditional probability $\mathrm{P}_{2}(1 / 2)$ has a simple interpretation. The analogue of (2.3) may be written as

$\int d I_{1} \int d v_{1} P_{1}(1) P_{2}(1 \mid 2)=P_{1}(2)$

and from this we infer, that $\mathrm{P}_{2}(1 / 2)$ is the kernel function of an evolution operator, which solves the transfer problem for $\mathrm{P}_{\uparrow}$.

In order to construct $\mathrm{P}_{2}(1 \mid 2)$ we approximate $k(x, v(x)), S(x, v(x))$ and $\mathrm{v}(\mathrm{x})$ by step-functions. Then we consider one arbitrary realization of the velocity field between $x_{1}$ and $x_{2}$ with fixed velocities $v_{1}$ at $x_{1}$ and $v_{2}$ at $x_{2}$. If the final intensity at $x_{2}$ is just equal to $I_{2}$, the initial intensity $I_{1}$ at $x_{1}$ is given by

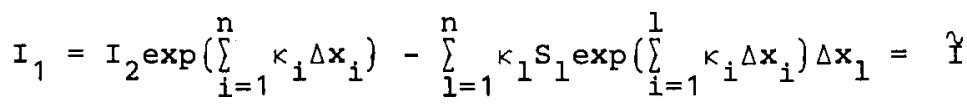

with $k_{i}=k\left(x_{i}, v\left(x_{1}\right)\right), S_{i}=S\left(x_{i}, v\left(x_{i}\right)\right)$ and $\Delta x_{i}$ beeing the length's of the intervals of the step-functions. This is simply a discretized version of the solution of the ordinary equation of radiative transfer. Then $\mathrm{P}_{2}(1 / 2)$ is given by 


$$
\begin{gathered}
P_{2}(1 \mid 2)=\int d v_{\alpha_{1}} \ldots \int d_{\alpha_{n-1}}\left(\mathbb{P}_{n+1}\left(1, \alpha_{1}, \ldots, \alpha_{n-1}, 2\right) / \mathbb{P}_{1}(1)\right) \exp \left(\sum_{i=1}^{n} k_{i} \Delta x_{i}\right) \cdot \\
\delta\left(I_{1}-\widetilde{I}\right) .
\end{gathered}
$$

The delta function assures, that only those realizations of the velocity field contribute, which have the correct final intensity $I_{2}$ for fixed $I_{1}$. The factor $\mathbb{P}_{n+1} / \mathbb{P}_{1}$ is the probability of realization of the considered step-function approximation of the velocity field. The exponential function takes care of the contraction of the interval $\mathrm{dI}_{1}$ to $\mathrm{dI}_{2}$ in going from $x_{1}$ to $x_{2}$. Finally, we integrate over all possible velocities at the division points of the interval $x_{1}, x_{2}$. More details with respect to the derivation of $\mathrm{Eq} .(3.7)$ can be found in paper I.

\section{The Mean Intensity and the Conditional Intensity}

In many cases, interest is concentrated on the mean intensity 〈I〉. This quantity can be calculated from $P_{1}$ as follows:

$\langle I\rangle=\int \mathrm{dv} \int \mathrm{d} I \cdot I \cdot \mathrm{P}_{1}$

The direct calculation of $\langle I\rangle$ or $\mathrm{P}_{1}$ may become quite tedious. However, for the quantity

$Q=\int d \cdot I \cdot P_{1}$

one derives from (3.5) and (3.6) an equation, which can often be solved much easier than the equation for $\langle I\rangle$ or $P_{1}$. The mean intensity $\langle I\rangle$ is obtained from $Q$ by a simple integration.

In order to derive the equation for $Q$, one multiplies (3.7) with $I_{2}$ and integrates with respect to $\mathrm{I}_{2}$ with the result

$$
\begin{gathered}
Q(2)=\int d v_{1} \int d v_{\alpha_{1}} \ldots \int d v_{\alpha_{n-1}}\left(\mathbb{P}_{n+1}\left(1, \alpha_{1}, \ldots, \alpha_{n-1}, 2\right) / \mathbb{P}_{1}(1)\right) \exp \left(-\sum_{i=1}^{n} k_{i} \Delta x_{i}\right) \cdot \\
\left\{Q(1)+\sum_{l=1}^{n} k_{1} s_{1} \exp \left(\sum_{i=1}^{1} \kappa_{i} \Delta x_{i}\right) \mathbb{P}_{1}(1) \Delta x_{1}\right\} .
\end{gathered}
$$

Since one easily shows (of paper I): 
$\lim _{n \rightarrow \infty} \exp \left\{ \pm \sum_{i=1}^{n} k_{i} \Delta x_{i}\right\}=1+\sum_{\mu=1}^{\infty}(-1)^{\mu} \int_{x_{1}}^{x_{2}} d s_{1} \int_{s_{1}}^{x_{2}} d s_{2} \ldots \int_{s_{n-1}}^{x_{2}} d_{n_{\nu=1}}^{\mu} k(\nu)$,

one arrives at the final equation

$Q(2)=\int \mathrm{dv}, Q(1) \mathbb{E}(1,2)+\int_{x_{1}}^{x_{1}} \mathrm{ds}_{\alpha} \int \mathrm{dv}_{\alpha} \mathbb{P},(\alpha) \kappa(\alpha) \mathrm{s}(\alpha) \mathbb{E}(\alpha, 2)$

with

$$
\begin{aligned}
\mathbb{E}(1,2)=\mathbf{P}_{2}(1 \mid 2)+ & \sum_{n=1}^{\infty}(-1)^{n} \int_{x_{1}}^{x_{2}} d s_{\alpha_{1}} \ldots \int_{s_{\alpha_{n-1}}}^{x_{2}} s_{\alpha_{n}} \int d v_{\alpha_{1}} \ldots \int d v_{\alpha_{n}} . \\
& \left(\mathbb{P}_{n+2}\left(1, \alpha_{1}, \ldots, \alpha_{n}, 2\right) / \mathbb{P}_{1}(1)\right) \sum_{\mu=1}^{n} \kappa\left(\alpha_{\mu}\right) .
\end{aligned}
$$

Eqs. (4.5) and (4.6) are the basic equations, which serve to calculate the mean intensity for arbitrary velocity fields, described by their n-point probability densities.

\section{Stochastic Models}

Different stochastic models have been used to treat the line transfer problem in presence of velocity fields. These models are discussed in some detail in the preceding contribution of Traving. Thus we limit ourselves at this place to show, how they fit into our general formalism.

a) The classical microturbulence-macroturbulence approach

Pure microturbulence is described by n- point probability densities of the type (2.10). Pure macroturbulence on the other hand is described by $\mathbb{P}_{n}$ 's of the type (2.6). The superposition of both yields n-point probability densities of the type

$\mathbb{P}_{\mathrm{n}}\left(\left(\mathrm{v}_{1}, \ldots, \mathrm{v}_{\mathrm{n}}\right)=\int \mathrm{d} \mathrm{w}_{1} \mathbb{P}_{1}^{\mathrm{mac}}\left(\mathrm{w}_{1}\right) \prod_{i=1}^{\mathrm{n}} \mathbb{P}_{1}^{\mathrm{mic}}\left(\mathrm{v}_{i}-\mathrm{w}_{1}\right)\right.$

Then, a simple calculation shows that the mean value <I> is just 


$$
\begin{aligned}
\langle I\rangle=\int d w_{1} \mathbb{P}_{1}^{\operatorname{mac}}\left(w_{1}\right) & {\left[I_{0} \exp \left(-\int_{x_{1}}^{x_{2}} d s k_{\operatorname{mic}}\left(w_{1}\right)\right)+\right.} \\
& \left.\int_{x_{1}}^{x_{2}} d s \exp \left(-\int_{s}^{2} d s^{\prime} k_{\operatorname{mic}}\left(w_{1}\right)\right) s\left(s^{\prime}\right) k_{\operatorname{mic}}\left(w_{1}\right)\right]
\end{aligned}
$$

where

$k_{\operatorname{mic}}\left(w_{1}\right)=\int d v \mathbb{P}_{1}^{m i c}(v) \kappa\left(v-w_{1}\right)$

and we have used the obvious initial condition

$Q(1)=I_{0} \mathbf{P}_{1}(1)$

Eq. (5.2) is just the classical microturbulence-macroturbulence result, as was to be expected.

b) Markov-processes

Markov-processes can be defined by the property

$\mathbb{P}_{n}(1, \ldots, n) / \mathbb{P}_{n-1}(1, \ldots, n-1)=\mathbb{P}_{2}(n-1 \mid n)$

Then the general $\mathbb{P}_{n}$ can be expressed by $\mathbb{P}_{2}(i-1 \mid i)$ as follows:

$\mathbb{P}_{\mathrm{n}}(1, \ldots, \mathrm{n})=\mathbf{P}_{1}(1) \prod_{i=2}^{\mathrm{n}} \mathbf{P}_{2}(i-1 \mid i) \quad$.

The conditional probability $\mathbb{P}_{2}(i-1 \mid i)$ is due to condition (2.3) subject to the restriction to be a solution of

$\int d v_{2} \mathbb{P}_{2}(1 \mid 2) \mathbb{P}_{2}(2 \mid 3)=\mathbb{P}_{2}(1 \mid 3)$

Examples of $\mathbb{P}_{2}$ are given in the contribution of Traving. For other examples see for instance Brissaud and Frisch (1974).

While pure microturbulence can be interpreted as a stochastic process without memory on the velocities encountered at $x_{i}$ if we go from $x_{i}$ to $\mathbf{x}_{i+1}$, the Markov-process is a stochastic process with "short" memory. The velocities encountered at $x_{i+1}$ are not independent of the velocity. which we have found at $x_{1}$, but are completely uncorrelated with all pre- 
vious velocities at $x_{j}$ with $j<i$.

In the case of Markov-processes, a simple equation for $Q$ can be derived. By multiplying Eq.(4.6) with $k(0) \mathbb{P}_{2}(0 \mid 1)$ and integrating with respect to $v_{0}$ and $x_{0}$, one derives the following integral equation for $\mathbb{E}$ (see paper I) :

$\int_{\mathbf{x}_{1}}^{\mathbf{x}_{2}} d s_{1} \int d v_{1} k(1) \mathbb{P}_{2}(1 \mid 2) \mathbb{E}(0,1)=-\mathbf{E}(0,2)+\mathbb{P}_{2}(0 \mid 2)$

Then, by multiplying $(4.5)$ by $K(\alpha) \mathbb{P}_{2}(\alpha \mid 2)$ and integrating with respect to $v_{\alpha}, x_{\alpha}$ one derives by using $(5.8)$ :

$Q(2)=\int d v_{0} \mathbb{P}_{2}(0 \mid 2) Q(0)-\int_{x_{0}}^{x_{2}} d x_{1} \int_{-\infty}^{+\infty} d v_{1} k(1) \mathbb{P}_{2}(1 \mid 2)\left\{Q(1)-\mathbb{P}_{1}(1) s(1)\right\}$.

Differentiating this with respect to $x_{2}$, we obtain

$\frac{\partial Q(0)}{\partial x_{0}}=\int d v_{0}\left[\lim _{x_{1} \rightarrow x_{0}} \frac{\partial}{\partial x_{1}} \mathbb{P}_{2}(0 \mid 1)\right] Q(0)-k(0)\left\{Q(0)-\mathbb{P}_{1}(0) S(0)\right\}$

which is equivalent with equation (25) of the preceding contribution of Traving. For a discussion of the special model of Auvergne et al (1973) and Gail et al (1974) see that contribution.

c) Higher order models

The microturbulence model and the Markov-process model may be considered as the two lowest order members of a hierarchy of model equations in the following sense:

(i) The microturbulence model assumes, that the general $\mathbb{P}_{n}$ can be factorized into a product of one-point probability densities $\mathbb{P}_{1}\left(v_{i}\right)$.

(ii) The Markov-process model assumes, that the general $\mathbb{P}_{n}$ can be factorized into a product of two-point conditional probability densities $\mathbb{P}_{2}(i \mid i+1)$ (cf Eq. $\left.(5.6)\right)$.

(iii) The next step would be to assume, that the general $\mathbb{P}_{n}$ can be factorized into a product of three-point conditional probability densities $\mathbb{P}_{3}(i, i+1 \mid i+2)$ and to derive a model equation based on this special form of the $\mathbb{P}_{\mathrm{n}}$. 
In this way, one would obtain a hierarchy of model equations which allow to incorporate an increasing degree of information on the structure of the velocity field into the theory. However, higher order models have not been studied up to now.

\section{Some comments on the relevance of low-order model equations}

In this chapter, we consider velocity fields with finite correlation length. The starting point are Eqs. (4.5) and (4.6). From these one derives

$\left.\left\langle I\left(x_{2}\right)\right\rangle=S\left(x_{2}\right)-\int_{x_{1}}^{x_{2}} d t^{\prime}\left\langle E\left(x_{2}, t^{\prime}\right)\right\rangle \frac{d S\left(t^{\prime}\right)}{d t^{\prime}}+\left(I\left(x_{1}\right)-S\left(x_{1}\right)\right)<E\left(x_{2}, x_{1}\right)\right\rangle$

with

$\left\langle E\left(x_{2}, x_{1}\right)>=\exp \left\{-\int_{x_{1}}^{x_{2}} d t^{\prime} k_{2}\left(t^{\prime}\right)\right\}\left(1+\sum_{n=1}^{\infty}(-1)^{n} e_{n}\left(x_{2}, x_{1}\right)\right)\right.$,

where

$e_{n}\left(x_{2}, x_{1}\right)=\int_{x_{1}}^{x_{2}} d t_{1} \int_{x_{1}}^{t_{1}} d t_{2} \ldots \int_{x_{1}}^{t} d t_{n} \int_{-\infty}^{+\infty} d v_{1} \ldots \int_{-\infty}^{+\infty} d v_{n} \mathbb{P}_{n}(1, \ldots, n) \sum_{\mu=1}^{n} k(\mu)$

Here we have assumed, that the absorption coefficient consists of two parts

$\kappa=k_{1}(v)+k_{2}$,

one of which, $\kappa_{2}$, is independent of the velocity. The actual choice of $k_{1}$ and $k_{2}$ will be specified later.

a) The case $x_{2}-x_{1}<1$

At this place we choose

$\kappa_{1}=\kappa_{\text {line }}, \kappa_{2}=\kappa_{\text {continuum }}$.

We introduce the new integration variable $s_{i}=\left(t_{i}-x_{i}\right) / 1$. By assumption we have

$\varepsilon=\left(x_{2}-x_{1}\right) / 1 \ll 1$ 
It is natural, to expand the integrand in (6.3) into a Taylor series with respect to the small quantities $s_{1} \ldots, s_{n}$. Then all s-integrations are easily done and one obtains to first order in the small quantity $\varepsilon$

$$
\begin{gathered}
\mathbf{e}_{n}=1^{n} \int d v_{1} \ldots \int_{n} d v_{n}\left[\mathbb{P}_{1}(1) \prod_{\mu=2}^{n} \delta\left(v_{\mu-1}-v_{\mu}\right)+\left.\frac{\varepsilon}{n+1} \sum_{i=1}^{n} \frac{\partial \mathbb{P}_{n}}{\partial s_{i}}\right|_{0}(n+1-i)\right] \cdot \\
\qquad \int_{0}^{\varepsilon} d s_{1} \ldots \int_{0}^{s} \prod_{\nu=1}^{n-1} k(\nu) .
\end{gathered}
$$

The dominating contribution corresponds to pure macroturbulence, as was to be expected. The first order correction depends only on $\mathbb{P}_{3}$, since due to (2.6) and assuming $\mathbb{P}_{n}$ to be uniform continuous at the origin, we have

$$
\begin{aligned}
\left.\frac{\partial \mathbf{P}_{n}}{\partial \mathbf{s}_{i}}\right|_{0} & =\lim _{\mathbf{s}_{i+1} \rightarrow 0} \lim _{\mathbf{s}_{n}+\mathbf{s}_{i+1} \operatorname{sim}_{i-1} \rightarrow \mathbf{s}_{1}} \frac{\partial \mathbf{P}_{n}}{\partial \mathbf{s}_{i}}= \\
& =\lim _{\mathbf{s}_{i+1} \rightarrow 0} \frac{\partial \mathbf{P}_{3}(0, i, i+1)}{\partial \mathbf{s}_{i}} \prod_{\mu=2}^{i-1} \delta\left(v_{\mu-1}-v_{\mu}\right) \prod_{v=i+2}^{n}\left(v_{v-1}-v_{v}\right) .
\end{aligned}
$$

Since preliminary results indicate, that 1 is of the order of the skale height of the atmosphere (see the subsequent contribution of sedlmayr), the present case applies to strong lines. Thus, in strong lines no information on the structure of the velocity field is contained, which extends beyond the three-point probability density $\mathbb{P}_{3}$.

b) The case $x_{2}-x_{1} \gg 1$

At this place we choose

$$
\kappa_{\text {mic }}=\int d v_{1} P_{1}(1) \kappa_{\text {line }}(1)
$$

$\kappa_{1}=\kappa_{\text {line }}-\kappa_{\text {mic }}, \kappa_{2}=\kappa_{\text {continuum }}+\kappa_{\text {mic }}$.

The integration in (6.3) is extentended over a n-dimensional simplex. Within this volume, the quantity

$$
<k_{1}(1) \cdots k_{1}(n)>=\int d v_{1} \ldots \int d v_{n} \mathbb{P}_{n}(1, \ldots, n) k_{1}(1) \cdots k_{1}(n)
$$

is different from zero only in regions of the integration volume, where all points $s_{i}$ form clusters of at least two points with mutual distances between the members of a cluster of at most $\sim 1$ correlation length 1 . If 
at least one point is isolated, then due to $(2.8)$ there will occur a factor $\left\langle k_{1}\right\rangle$ in $(6.11)$ which is zero according to the definition of $k_{1}$. By analyzing the various possible clusters, one can show that, provided the condition

$\max k_{1}(v) \cdot 1 \ll 1$

$\forall \mathrm{v}$

is satisfied (cf Brissaud and Frisch, 1974, and Frisch,1968), the dominating contribution is provided by two cases: (i) only clusters of two points occur and (ii) besides one or at most 2 clusters of three points only clusters of pairs occur. Since $\max \left(k_{1}(v)\right)$ is of the order of the line absorption coefficient in the centre of the line, this case corresponds to weak lines, since 1 itself is probably of the order of the scale height. Thus, weak lines, just as strong lines, do not contain any significant information on the structure of the velocity field extending beyond $\mathbb{P}_{3}$.

These results suggest, that model equations for the radiative transfer problem in moving media based on $\mathbb{P}_{2}$ or $\mathbb{P}_{3}$ are sufficient, at least for strong and weak lines.

\section{Acknowledgement}

This work has been performed as part of the program of the Sonderforschungsbereich 132 "Theoretische und praktische stellarastronomie" which is sponsored by the Deutsche Forschungsgemeinschaft. 